\title{
Weight loss may be unrelated to dietary intake in the imiquimod-induced plaque psoriasis mice model
}

https://doi.org/10.1515/biol-2020-0009

Received January 22, 2019; accepted November 26, 2019

Keywords: imiquimod, plaque psoriasis, weight loss, food intake, water intake, moderate hypothermia

Since 2009, the imiquimod (IMQ)-induced mice model has been extensively adopted as a model of human plaque psoriasis to investigate the mechanisms of psoriatic pathogenesis and to verify the effective of anti-psoriatic drugs [1]. However, the side effects and the mechanism of IMQ-induced weight loss have not been investigated. Recently, we focused on some of those issues in an article which claimed that IMQ induced weight loss and decreased food intake of $\mathrm{C} 57 \mathrm{BL} / 6 \mathrm{~J}$ mice in the model of generalized pustular psoriasis [2]. The difference of the two mice model between the generalized pustular psoriasis and the plaque psoriasis is the time taken to induce by IMQ. The former is induced for 4 consecutive days, while the latter is induced for 7 consecutive days. Therefore, we were interested in whether IMQ caused weight loss and the mechanism behind plaque psoriasis in the mice model.

The male BALB/mice (6-8 weeks of age) were purchased from the Center of Laboratory Animals of Southern Medical University (Guangzhou, China). The mice were randomly divided into two groups of 5-6 each.

*Corresponding author: Wanqun Chen, Department of Gastroenterology, Chongqing Hospital of Traditional Chinese Medicine, 6 panxi branch road, Chongqing 400021, China. Tel: $+86-$ 023-67063949. Email: cwq20130219@163.com

Jinwei Zhang, Department of Dermatology and Cosmetology, Chongqing Hospital of Traditional Chinese Medicine, Chongqing 400021, China

Jinwei Zhang, College of the Second Clinical Medicine, Guangzhou University of Chinese Medicine, Guangzhou 510006, China

Xiaojun Yang, Hong Qiu, Wanqun Chen, Department of Gastroenterology, Chongqing Hospital of Traditional Chinese Medicine, Chongqing 400021, China

\#These authors have contributed equally to this work.
After 1 week of adaptive feeding, the back hair $(1.5 \times 1.0$ $\mathrm{cm})$ of the mice was shaved. After 2 days, the two groups of mice were treated topically with vehicle (Vaseline, Lierkang Medical Technology Co., Ltd., Shandong, China; $62.5 \mathrm{mg}$ per mouse) or vehicle and IMQ cream (Ming Xin Pharmaceutical Co. Ltd., Sichuan, China; $62.5 \mathrm{mg}$ per mouse) on their shaved back skin for 7 consecutive days.

The psoriasis area and severity index (PASI) scores were monitored to confirm the successful establishment of plaque psoriasis. An objective scoring system was developed based on the clinical PASI to score the severity of mice skin lesion. The scaling, thickening and erythema was scored on a scale from 0 to 4 ( 0 represented none; 1 represented slight; 2 represented moderate; 3 represented marked; 4 represented very marked). The cumulative scores (scaling plus thickening plus erythema) served as a measure of the severity of lesions (scale $0-12$ ). The single-blind way was used for the PASI scores. Mice on the 2nd day after IMQ induction exhibited signs of plaque psoriasis-like lesion began to appear such as scaling, thickening, erythema and the severity of lesion raised over time (Figure 1A-D). In contrast, the mice treated topically with Vaseline displayed no scaling, thickening or erythema. Those results were consistent with previous study [1] and it suggested that the mice model of plaque psoriasis was successfully established.

Subsequently, we investigated whether IMQ caused weight loss of mice in the model of plaque psoriasis. Mice were weighed by electronic scale from the 1st day to the 7th day. Results show (Figure 2A) IMQ caused weight loss of BALB/c mice from the 2nd day to the 7th day. As diet is the determining factor affecting weight, the amount of food and water intake of mice was weighed throughout the trial (We changed fresh food and water, then weigh them to calculate intake at 9 am every day). There was a reduction of food intake caused by IMQ from the 1st day to the 4th day, v-shaped, and this was most evident on the 2nd day (Figure 2B). The food intake of mice induced by IMQ tended to increase compared to the control group 
$\mathbf{A}$

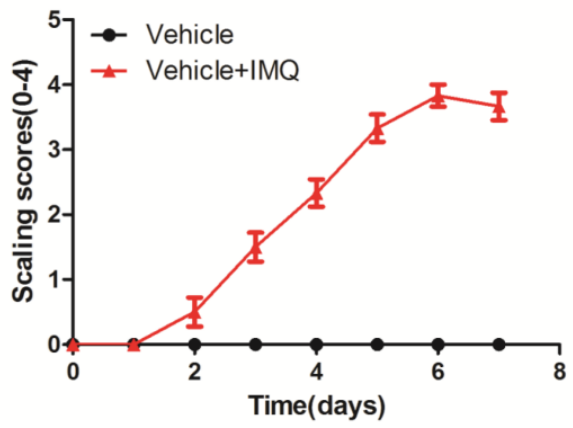

C

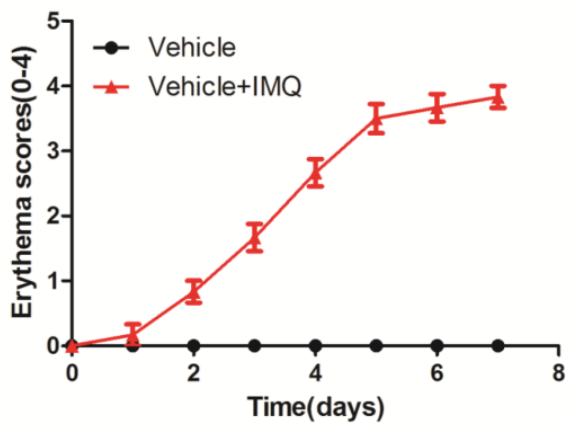

B

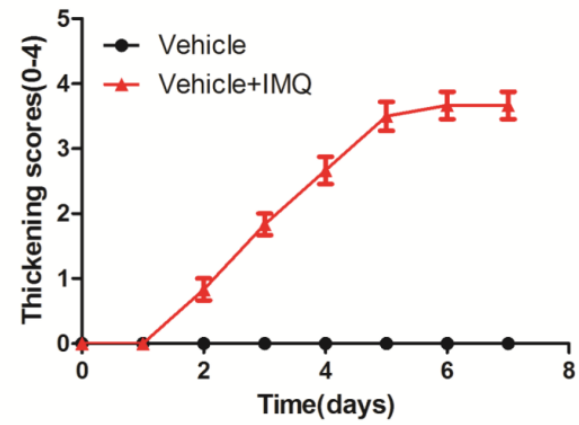

D

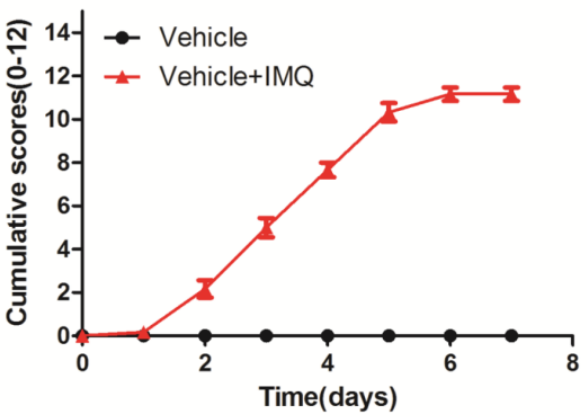

Figure 1. PASI scores of the mice model of plaque psoriasis. Male BALB/c mice were treated with vehicle or vehicle and IMQ cream on their shaved back skin for 7 consecutive days. Scaling (A), thickening (B), erythema (C) and cumulative (D) scores of the skin lesion. Data was shown as means \pm SEM (5-6 mice per group, $n=3)$. Scaling, thickening and erythema were scored respectively on a scale from 0-4 (0, none; 1 , slight; 2 , moderate; 3 , marked; 4 , very marked). Cumulative scores were represented by scaling scores plus thickening scores plus erythema scores.

A

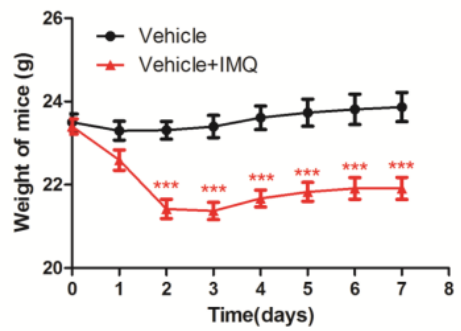

B

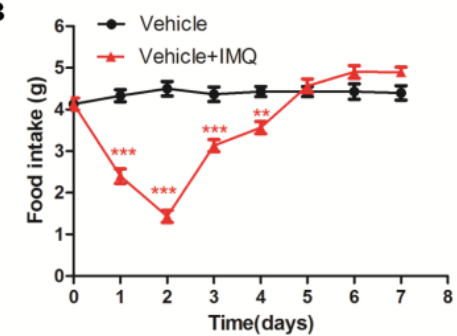

C

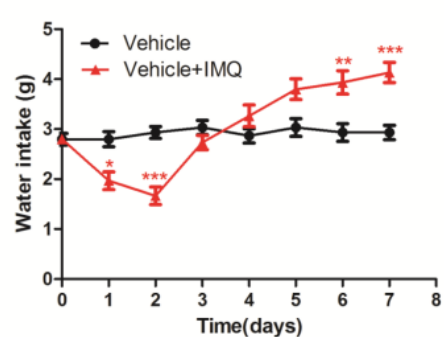

Figure 2. IMQ treatment causes sustained weight loss, but increased appetite after appetite loss in the mice model of plaque psoriasis. Male $\mathrm{BALB} / \mathrm{c}$ mice were treated with vehicle or vehicle and IMQ cream on their shaved back skin for 7 consecutive days. Weight (A), food intake (B) and water intake (C) of the mice. Data was shown as means \pm SEM (5-6 mice per group, $\mathrm{n}=3$ ) and analyzed by t-test. * represents $P<0.05,{ }^{\star *}$ represents $P<0.01$ and $* \star \star$ represents $P<0.001$, compared with vehicle at individual timepoints.

from the 5 th day to the 7 th day (Figure 2B). On the other hand, the water intake of these mice was reduced from the 1st day to the 3rd day, and was at the lowest on the 2nd day. This followed a similar to the trend of the food intake (Figure 2C). There was an increase in the water intake of mice induced by IMQ compared to the control between the 6 th day to the 7 th day (Figure $2 \mathrm{C}$ ).

To further investigate the relationship of weight loss and food or water intake, we calculated the linear relationship between them. We found that there was no obvious relationship identified between weight loss and diet (Figure 3A and B). However, food intake was positively correlated with water intake (Figure 3C). These results suggest that weight loss in this model was independent of food and water intake. Clearly, the mechanism of weight loss was different between the model of plaque psoriasis and generalized pustular psoriasis induced by IMQ. Thus, further study is required to deduce the reason of weight loss of the mice in this model of plaque psoriasis.

We noted a case report that patients had systemic side effects when using IMQ to treat basal cell carcinoma [3]. The patient exhibited weight loss, loss of appetite, and 
flu-like symptoms following treatment. However, in our work we found IMQ induced BALB/c mice weight loss and increased appetite only on the 7th day. We conclude the reason behind why loss of appetite occurred in the former while an increase of appetite in the latter was due to the flu-like symptoms. Therefore, we hypothesized that body temperature may have affected the appetite of the mice. We examined the body temperature of mice from the the 1st day to the 7th day by non-contact infrared clinical thermometer (Shenzhen Ailikang Medical Co., Ltd., Shenzhen, China). The body temperature of the mice in the IMQ-induced group was significantly lower than that in the normal group from the 4th day to the 7th day (Figure 4). To further investigate the relationship of body temperature to food or water intake, we calculated the linear relationship with them. The results showed body temperature was negatively correlated with food or water intake (Figure 5A and B). Thus, it may be moderate hypothermia causing the increased appetite in order to provide more energy to the mice.

In our study we identified that in the process of IMQ-induced plaque psoriasis mice model, this resulted in decreased body weight, with the appetite of the mice decreased at first then increasing. We believe that this

$\mathbf{A}$

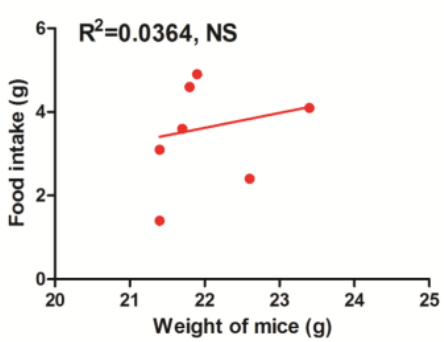

B

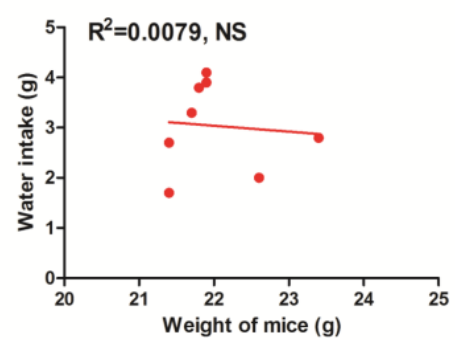

weight loss is independent of food or water intake. The increase in food and water intake may be because of the moderate hypothermia induced by IMQ. These findings may be of benefit to elucidate the mechanism of the mice model of plaque psoriasis.

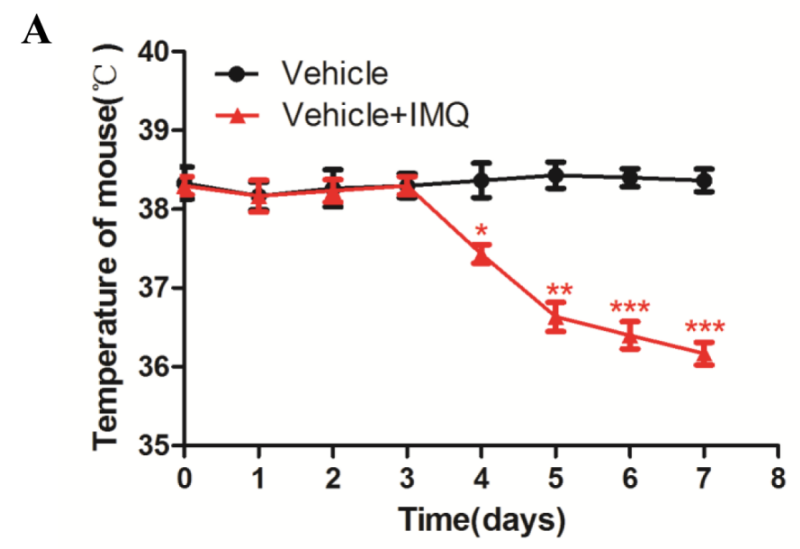

Figure 4. Body temperature of the mice. Male BALB/c mice were treated with vehicle or vehicle and IMQ cream on their shaved back skin for 7 consecutive days. Body temperature of mice from the 1st day to the 7th day was tested by non-contact infrared clinical thermometer. Data shown as means \pm SEM (5-6 mice per group, $\mathrm{n}=3$ ) and analyzed by t-test. * represents $P<0.05$, ** represents $P<0.01$, ** represents $P<0.001$, compared with vehicle.

C

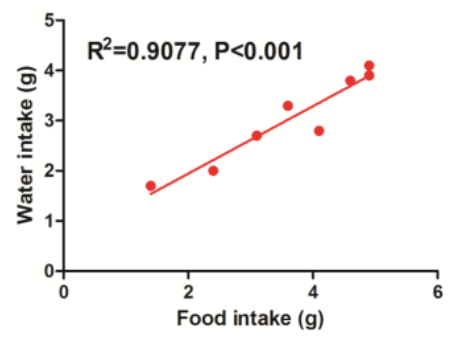

Figure 3. Weight loss of the mice is independent of food or water intake in the model of plaque psoriasis. Linear relationship between weight loss and food intake (A), weight loss and water intake (B) and food intake and water intake (C). Data was shown as means \pm SEM (5-6 mice per group, $\mathrm{n}=3$ ) and analyzed by corelation analyze.

A

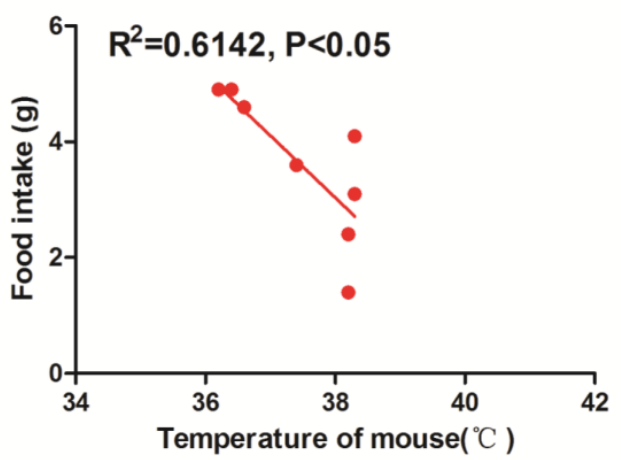

B

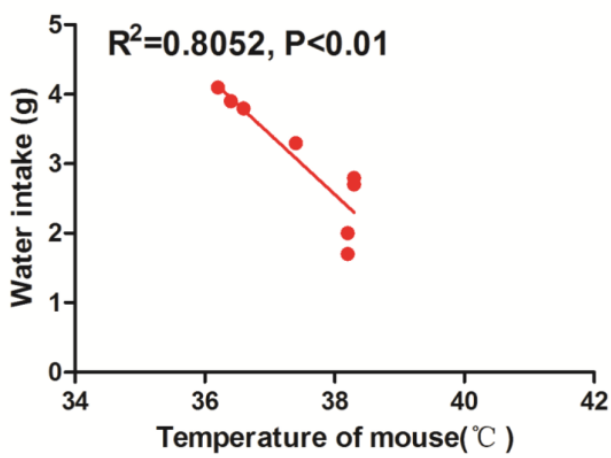

Figure 5. Increased appetite is caused by moderate hypothermia in the model of plaque psoriasis. Linear relationship between body temperature and food intake $(A)$ and body temperature and water intake (B). Data shown as means \pm SEM (5-6 mice per group, $n=3)$ and analyzed by 
Ethical approval: The research related to animals use has been complied with all the relevant national regulations and institutional policies for the care and use of animals. All the experiments were in compliance with the National Institutes of Health Guide for the care and use of laboratory animals with the approval of Animal Care and Use Committee of Chongqing Hospital of Traditional Chinese Medicine.

Acknowledgements: This work was funded by National Natural Science Foundation of China (81904175), Chongqing Performance Reword Foundation (cstc2017jxj1130019), Chongqing Science and Technology Project (cstc2018jcyjAX0756) and Chongqing Health Planning Commission Project (ZY201802063).

Conflict of interest: Authors state no conflict of interest.

\section{References}

[1] van der Fits L, Mourits S, Voerman JS, Kant M, Boon L, Laman JD, et al. Imiquimod-induced psoriasis-like skin inflammation in mice is mediated via the IL-23/IL-17 axis. Journal of immunology. 2009;182:5836-5845.

[2] Alvarez P, Jensen LE. Imiquimod Treatment Causes Systemic Disease in Mice Resembling Generalized Pustular Psoriasis in an IL-1 and IL-36 Dependent Manner. Mediators of inflammation. 2016:6756138.

[3] Hanger C, Dalrymple J, Hepburn D. Systemic side effects from topical imiquimod. The New Zealand medical journal. 2005;118:U1682. 